\title{
The Origin of the Earth-Moon System as Revealed by the Moon
}

\author{
Primary Authors:
}

Rufu, R. - Southwest Research Institute (raluca@boulder.swri.edu)

Salmon, J.- Southwest Research Institute (julien@boulder.swri.edu)

Co-Authors: Kaveh Pahlevan (SETI Institute), Channon Visscher (Space Science Institute and Dordt University), Miki Nakajima (University of Rochester), Kevin Righter (NASA Johnson Space Center).

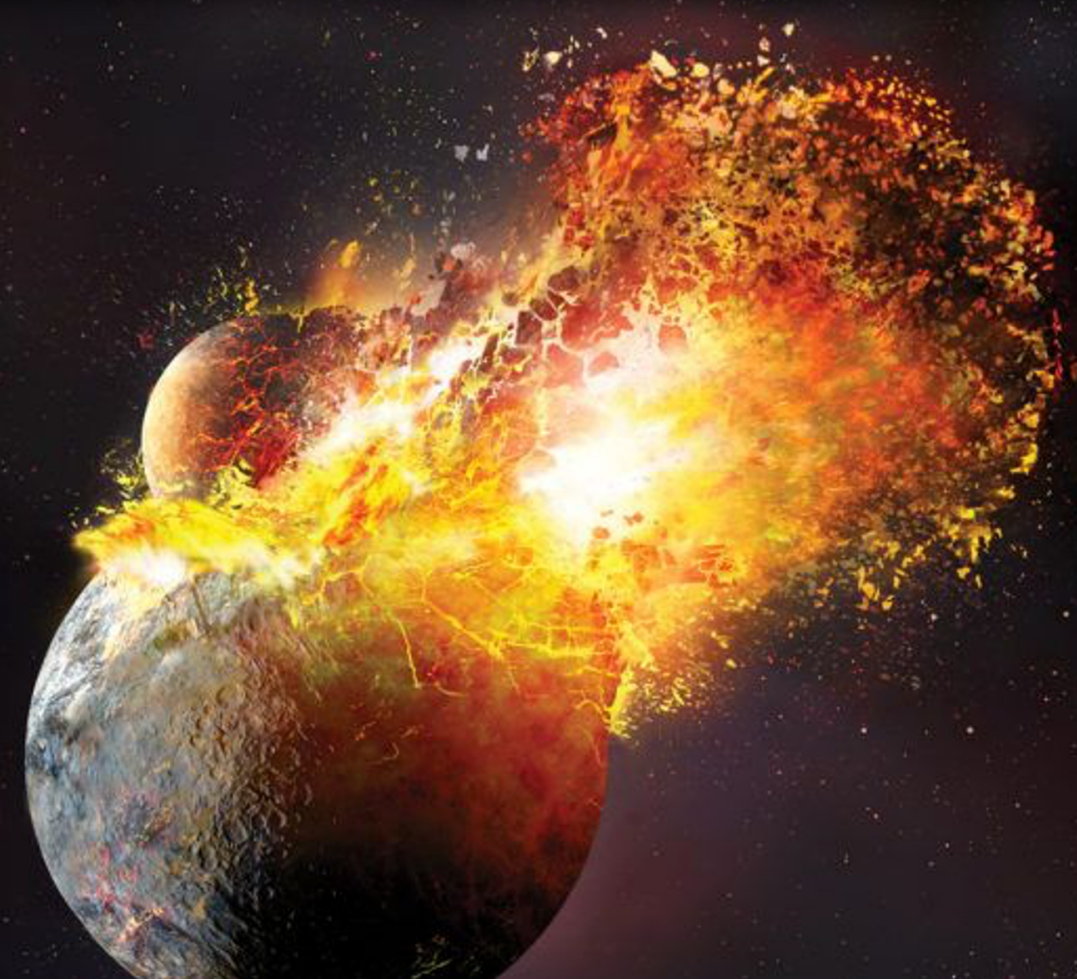

Pic: Dana Berry

Endorsers: Francis Nimmo (UC Santa Cruz), Sarah T. Stewart (UC. Davis), Simon Lock (Caltech), Clive Neal (Notre Dame), Oded Aharonson (PSI), Juliane Gross (Rutgers University), William Bottke (SwRI), Christian Koeberl (University of Vienna), Qing-zhu Yin (UC Davis), Stephen Elardo (University of Florida), Rachel Klima (Johns Hopkins), David S. Draper (NASA), Cari Corrigan (Smithsonian Institution), Kip Hodges (Arizona State University), Allan H. Treiman (USRA), Audrey Bouvier (Universität Bayreuth), Dustin Trail (University of Rochester), Wim van Westrenen (Vrije Universiteit Amsterdam), Rosalind Armytage (Jacobs-JETS, NASA), Douglas Currie (University of Maryland), Mahesh Anand (Open university UK), Carle M. Pieters (Brown University), Georgiana Kramer (PSI), Mark Robinson (Arizona University), Dimitri A. Papanastassiou (Caltech), Steven Simon(University of New Mexico), Katherine Shirley (University of Oxford.), Youxue Zhang (University of Michigan), James W. Head, Brown University, Jennifer Grier (PSI), Bernard Foing (ESA/ESTEC), Laurent Sibille (SURA), Tomas Magna, Czech Geological Survey, Cynthia Evans (NASA), Kevin D. McKeegan (University of California) Melissa Roth (LLC), Vince Roux (LLC), G. Jeffrey Taylor (University of Hawaii), Patricia W. Dickerson (University of Texas). Richard C. Greenwood (The Open University, UK) 


\section{Key Points:}

- Study of the Moon's origin is a potentially powerful way to constrain the end stages of terrestrial accretion.

- In the past decade, several Moon-forming impact scenarios have been proposed and continued investigation is needed to assess the feasibility of these scenarios.

- Samples from large lunar impact basins and advancements in modeling key processes are needed to further constrain lunar origin scenarios.

- Lunar seismic arrays would provide fundamental constraints on the interior structure, volatile content and formation history of the Moon.

\section{Introduction}

The leading theory for lunar origin is formation via a giant impact with the early Earth. Giant impacts are thought to be a ubiquitous feature of the later stages of terrestrial planet formation. Hence, understanding the Moon's origin is a potentially powerful way to constrain the end stages of terrestrial accretion, due to abundant lunar samples and the detailed compositional constraints they provide. However, the nature of this event remains highly debated. In the past decade, various Moon-forming impact scenarios have been proposed that invoke very different impact conditions. Assessing the feasibility of these scenarios and ultimately distinguishing between them is crucial to unraveling what our Moon can tell us about our planet's origin and earliest evolution, and the types of impacts typical during late stage terrestrial accretion.

\section{The "isotopic crisis" and the still open question of Moon formation}

The giant impact theory, first proposed in the mid-1970s [1-2], envisions that the Moon formed from a disk of ejecta produced by a collision with the Earth. Origin via impact became the leading hypothesis subsequent to the 1984 "Origin of the Moon" conference, based primarily on its perceived strength in accounting for Earth's rapid early spin, the Moon's lack of iron, and the initial lunar magma ocean [3], and the weaknesses of alternative formation theories. The first generation of 3D hydrodynamic impact simulations established that many giant impacts produced iron-poor, Earth-orbiting disks [4-7]. Later works showed that the current Earth-Moon angular momentum $\left(L_{\mathrm{EM}}\right)$, as well as the Moon's mass and bulk density, could be explained by a low-velocity, oblique impact by a Mars-sized body [8-10], in what has become known as the canonical Moon-forming impact.

In the past decade, the nature of a Moon-forming impact has become highly debated, due to the difficulty in explaining isotopic similarities between the Moon and Earth's mantle. A disk produced by a canonical impact originates mostly $(\geq 80 \%)$ from the outer layers of the impactor, rather than from the proto-Earth's mantle. A Moon accreted from such a disk would then have a composition similar to that of the impactor (Fig.1-a). Isotopic variations across meteorites from Mars and the asteroid belt, in combination with radial-mixing expected during terrestrial accretion, imply that the impactor would have been isotopically different than the Earth [11,12]. Yet, high-precision measurements show that the silicate Earth and Moon are isotopically indistinguishable across many elements $[13,14]$. Thus, while a canonical impact accounts for the angular momentum (AM) in the Earth-Moon system, it appears inconsistent with Earth-Moon compositional similarities.

\section{Alternative Moon-forming scenarios}

These results have motivated over the past decade a diverse array of impact scenarios (Table 1) that strive to better account for Earth-Moon isotopic similarities. 
Table 1: Current impact scenarios (References in superscript)

\begin{tabular}{|c|c|c|}
\hline Model Name & Impactor Mass $\left[\mathbf{M}_{\oplus}\right]$ & Impact velocity [ $\left.\mathbf{V}_{\text {esc }}\right]$ \\
\hline 1) Canonical ${ }^{8-10}+$ Earth-like Theia' ${ }^{15,16}$ & $0.13-0.2$ & $1-1.2$ \\
\hline 2) Canonical $^{8-10}+$ equilibration $^{12}$ & $0.13-0.2$ & $1-1.2$ \\
\hline 3) High-AM: Fast-spinning Earth ${ }^{17,18}$ & $0.03-0.1$ & $1.5-3$ \\
\hline 4) High-AM: Half-Earth ${ }^{18,19}$ & $0.4-0.5$ & $1-1.5$ \\
\hline 5) High-AM/-Energy + equilibration ${ }^{18}$ & $0.03-0.5$ & $1-3$ \\
\hline 6) Hit-and-run ${ }^{20}$ & $0.2-0.3$ & $1.2-1.4$ \\
\hline 7) Multiple-impact ${ }^{21}$ & $0.01-0.1$ & $1-3$ \\
\hline
\end{tabular}

Scenarios 1-2: Modified canonical impacts. Recent work [15] implies a substantial likelihood that Theia had an Earth-like isotopic composition in most elements (Fig.1-b). Initially it was argued that this might have been likely if Theia grew near 1 AU from the same building material as the Earth itself $[13,22]$. This notion seemed plausible because canonical impacts required Theia to have a low relative velocity at infinity, consistent with an Earth-like impactor orbit [8-9]. However, Pahlevan \& Stevenson [12] used impact statistics from an N-body planet accretion model and assumed that $\Delta^{17} \mathrm{O}$ is linearly correlated with the distance from the Sun, to estimate that the probability of Theia being appropriately Earth-like in $\mathrm{O}$ was only of order a percent. Additionally, new data indicated that the Earth and Moon had equal initial tungsten isotopic compositions [23-25], compounding the isotopic crisis. Theia's core and mantle W isotopic compositions would have been sensitive to the timing and conditions of its core's formation. Thus, even if Theia were Earth-like in elements like $\mathrm{O}$, by virtue of having formed near Earth, an additional coincidence would be needed for Theia's W composition to be consistent with an Earth-Moon W match.

Alternatively, an initially non-Earth-like disk may have become Earth-like as it diffusively mixed with vaporized portions of Earth's mantle [16]. Such an "equilibration" process is appealing because it could account for a wide range of Earth-Moon isotopic similarities, including W [16]. But whether the mixing processes would operate sufficiently in the immediate post-impact conditions before the Moon forms remains unclear [87].

Scenarios 3-5: High-AM impacts. Historically, the AM of the Earth-Moon system was assumed to have remained essentially constant over the age of the solar system, hence impacts focused on impact regimes that left the Earth-Moon system with an AM comparable to $L_{\mathrm{EM}}$. The "isotopic crisis" inspired exploration of other impact regimes, primarily by relaxing the AM constraint. High AM impacts produce a planet and protolunar disk with $\approx$ identical compositions for most elements even if the impactor was isotopically non-Earth like $[17,19]$. Lock et al. [18] further argued that mixing may yield similar disk-planet compositions across a broad range of high-AM impacts that produce vaporized, co-rotating "synestias" (Fig.1c). However, these impacts introduce a new challenge: excess AM delivered by the impact must somehow then be removed. Dynamical interactions with the Sun could have transferred substantial AM from the Earth-Moon system to Earth's heliocentric orbit after the Moon formed [17, 26-31]. Successful cases consistent with a final AM near $L_{\mathrm{EM}}$ were found but appeared to require a narrow range of tidal parameters. 


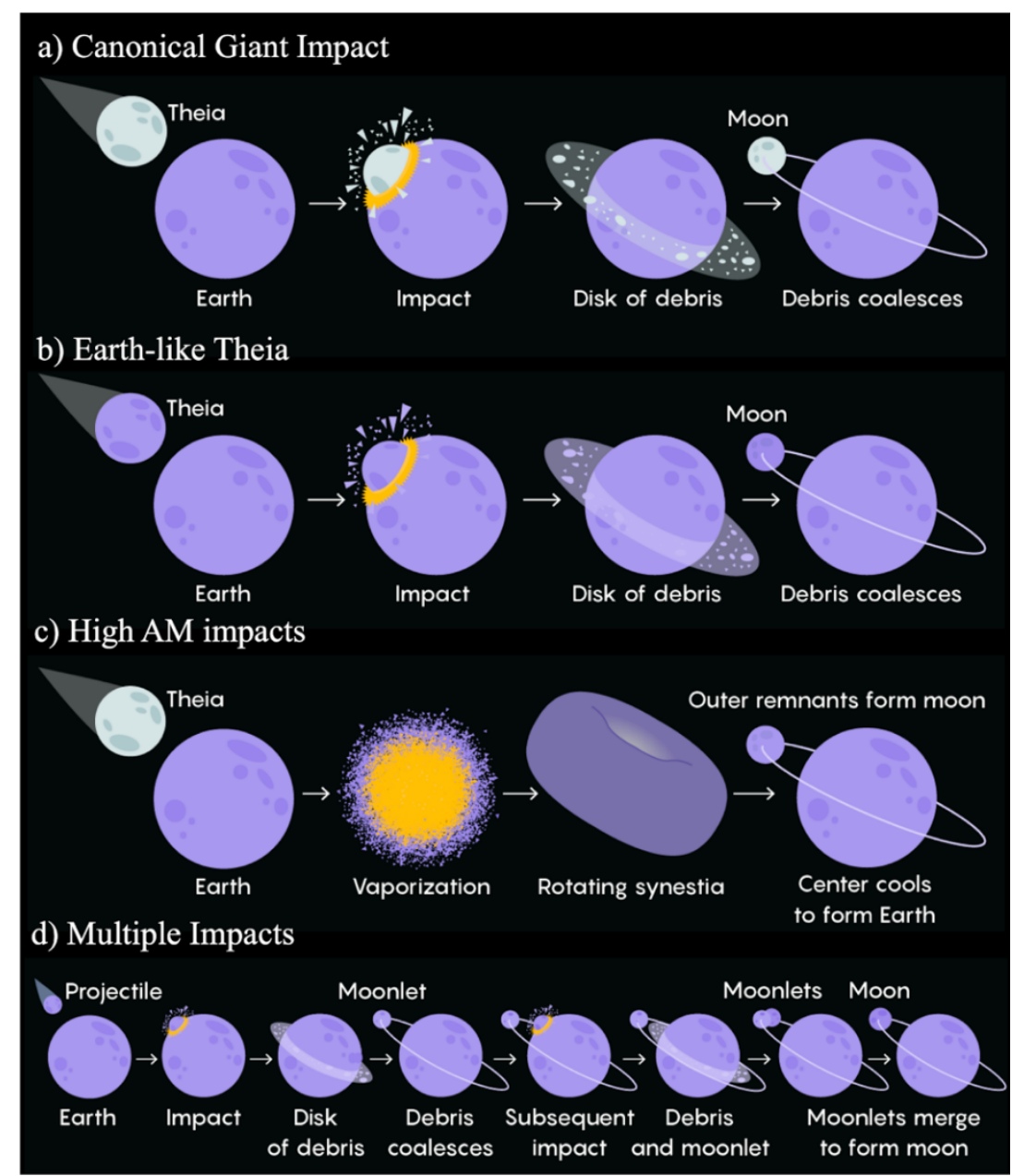

Fig. 1 - Schematics of different Moon-forming models. Credit: Lucy Reading-Ikkanda /Quanta Magazine

Scenario 6: Hit-and-run impact. A higher-velocity, less oblique impact produces a disk and planet that are more compositionally similar than in a canonical impact [20], requiring modest equilibration or a relatively Earth-like Theia. However it appears difficult for a single such collision to yield a massive enough disk.

Scenario 7: Multiple impacts. Rufu et al. modeled Moon formation via $\sim 20$ sub-Mars impactors [21]. Each impact creates a moonlet that tidally migrates outward. A later impact produces another moonlet, whose orbital expansion can cause it to merge with the prior outer moon (Fig.1-d). The Moon can then be built up by many impacts, with the final Earth-Moon compositions approaching that of the mean planetesimal neighborhood. However the merger efficiency between consecutive moonlets is low [32].

While these are many promising new ideas, the current scenarios face important challenges:

- Earth and Moon now appear to have had nearly equal W isotopic compositions, which argues for equilibration [16, 23-25]. But, current work finds only 0 to $\sim 50 \%$ of the Moon comes from the inner disk where Earth-disk mixing seems most likely $[18,33]$. The extent of post-impact mixing remains an open question, especially for the refractory isotopes such as titanium [14].

- Recent works argue that volatile escape to infinity is unlikely in scenarios (1) - (6) [34]. However, the lunar abundance and/or isotopic composition of some volatiles (e.g., Zn and 
perhaps $\mathrm{K}$; $[35-38,57])$ now appears to require some loss, at least from the lunar gravity well [39].

- Scenarios (3) - (5) produce synestias from which the Moon is predicted to form in $\sim 1 \mathrm{yr}$ [18], implying a fully molten Moon [40]. But, GRAIL data implies an only partially molten initial Moon [41,42].

- The likelihood of scenarios (3) and (7) depends on how multiple impacts affect planet rotation and moon retention, but this is uncertain, and current works reach contradictory conclusions [21, 32, 43, 89].

In summary, the Moon's origin is coupled to that of the Earth. Because of the Moon's accessibility, our knowledge of its detailed composition and physical properties will likely always exceed that of the other planets in the inner Solar System. Thus unraveling how the Moon formed holds the promise of providing a cornerstone for understanding how our terrestrial planets originated. However, realizing that promise has proved challenging. Current theoretical models provide an array of possible solutions, but great uncertainty remains in which is the most compelling explanation for our particular Earth-Moon system. The more than a half-dozen variants on the giant impact model described above span a wide range of impactor masses, and thus have very different implications for the final major event of Earth's accretion. In order to ultimately distinguish among this array of interesting theories, more data and physical constraints are needed.

\section{Key outstanding data needed to resolve lunar origin debate}

Over the past several decades, the efforts in modeling and analysis, combined with exploration missions, has greatly increased our understanding of lunar origin. But it has also opened a large variety of new questions and uncertainties. We here identify some of the important data needed to pierce the mystery of the Moon's origin.

- The Moon's initial thermal state. An apparent conflict exists between lunar formation models, which favor a hot start for the Moon, and geophysical constraints that imply an initial cool/solid lunar interior and a shallow magma ocean (MO; e.g., [44]). These constraints include the history of tectonics and strain [41, 45-47], observed crustal thickness compared to MO model predictions [42,48], and a seismic transition that may represent the $\mathrm{MO}$ base $[49,50]$. Alternative explanations for each observation exist [51], but it is unclear whether collectively these observations support a partially/fully molten initial Moon. A long-term seismic network and heat flow sensors could reveal new constraints on the depth of the initial lunar magma ocean. If, indeed the Moon cannot have been initially fully molten [42], this would provide a stringent constraint on lunar origin models [44], potentially ruling out those in which the Moon forms in $<<100 \mathrm{yr}[40]$.

- The lunar endogenic volatile content and its interpretation The Moon is depleted in volatile and moderately-volatile elements compared with Earth [52], but the cause of this remains poorly understood. Small enrichments in the heavy isotopes of some lunar volatile elements (e.g., Zn, K) have also been reported [35,36]. Unraveling how the Moon's volatile abundances originated could discriminate between different lunar origin models via differing predictions for relative volatile abundances or isotopic composition [39]. This could be achieved by additional analyses of lunar samples, gamma-ray and neutron spectrometer measurements (e.g., K/Th ratios) and acquisition of future samples that are more representative of the Moon's composition at depth (e.g., 
samples of the Moon's mantle potentially exposed at large impact basins such as South Pole Aitken, [53]). Current models for this depletion either invoke a viscous disk atmosphere [54,55], which would not permit efficient mixing with the Earth [56, 87] or a viscous melt layer [57], whose consequences for isotopic fractionation remain unexplored. The Moon's volatile content relative to Earth is a fundamental constraint. Further investigation is needed for a self-consistent explanation of the lunar volatile element depletion and heavy isotope enrichment, and its bearing on the relative roles of chemical processes during formation and early evolution.

- Additional high-precision measurements of the isotopic composition of lunar vs. terrestrial materials for highly refractory elements. A key uncertainty is whether vaporized portions of the protolunar disk could have mixed and compositionally equilibrated with the Earth. Given the complexity of the physics of equilibration, independent markers of its importance in establishing the Moon's bulk composition have been sought. Differences between the Earth and Moon isotopic compositions for highly refractory elements would seem to provide evidence for equilibration, because (at least) in vertically stratified disks [58-60], mixing would be restricted to elements in the vapor phase. Instead, the Earth and Moon have indistinguishable $\mathrm{Ti}$ compositions [14]. This does not, however, rule out equilibration, because Ti may have been partially vaporized [14], or refractory-rich droplets could have equilibrated with surrounding vapor, if the disk was vertically unstratified [61]. Recent high precision calcium data nominally imply a difference between the Earth and Moon Ca isotopic compositions, but it is small ( $<10 \mathrm{ppm}$; [62]). Additional high-precision measurements of refractory element isotopic compositions of lunar and terrestrial samples (including higher precision $\mathrm{Ca}$ data) would provide valuable new constraints on the likelihood and mechanism of equilibration.

- Determining the Moon's bulk composition. We currently lack direct samples of the Moon's mantle. Lunar material and the bulk silicate Earth (BSE) have similar major element abundances and nearly-identical isotopic compositions for, e.g., O, Si, and Ti, but the Moon is more depleted in volatiles as described above. Detailed Earth-Moon chemical relationships may ultimately provide a way to distinguish among lunar origin scenarios (Table 1), because those models will have varied pressure vs. temperature conditions of accreting lunar material that will affect the Moon's chemistry and isotopic abundances. Further, Pahlevan et al. [61] predicted that equilibration between the disk and the full terrestrial mantle would lead to a relationship between the resulting EarthMoon differences in $\mathrm{FeO} / \mathrm{MgO}$ and in silicon isotopic composition (with larger EarthMoon differences in $\mathrm{FeO} / \mathrm{MgO}$ implying larger differences in $\delta^{30} \mathrm{Si}$ ). Because the Earth and Moon are now known to have essentially identical Si compositions [63], equilibration thus implies that they should have similar $\mathrm{FeO} / \mathrm{MgO}$ as well, i.e., that the lunar $\mathrm{Fe} /(\mathrm{Fe}+\mathrm{Mg})$ ratio must be between 1 to 1.3 times that of the BSE [63]. Some prior estimates fell in this range $(\sim 1.2,[64,65])$, but more recent work including GRAIL results estimate this ratio is 1.4 [66-68], nominally inconsistent with equilibration. However, there are still substantial uncertainties that also permit a more BSE-like value. These issues could be better constrained by improved knowledge of the Moon's bulk composition (i.e., samples from diverse regions of the surface).

- Isotopic composition of Venus. Current knowledge of the isotopic composition of Mars heavily influences our thinking about the initial heterogeneity in the inner solar nebula. However, Mars's accretion was fast and its material locally sourced [69]. 
Knowledge of the isotopic composition of Venus, would provide a much stronger constraint for Earth, and provide a better understanding of the inner solar system mixing during accretion of the terrestrial planets. If the isotopic ratios (e.g., oxygen) of Venus proved similar to those in the Earth, the likelihood of an Earth-like impactor would be increased and the isotopic crises eased.

- Dating of the largest lunar basins. Progress regarding the isotopic diversity of Earthimpactors can also be made by observations that provide new constraints on early outer planet migration. Early migration during planet accretion [70, 71] would have tended to radially mix the inner Solar System, but if migration occurred later after planet accretion had ended $[72,73]$, the planets may have accreted in a compositionally more radially segregated disk. The timing of giant planet migration may be constrained by better knowledge of when the lunar basins formed. A broad dispersion of basin ages would suggest a decaying background population of impactors leftover from planet formation, consistent with early planet migration, while a clustering of late basin ages would more naturally support a later epoch of giant planet migration.

- Data that constrains the timing of magma ocean solidification. Explaining the Moon's current $5^{\circ}$ tilt relative to the ecliptic has been a longstanding issue for a giant impact origin, which would nominally produce a low-inclination Moon $\left(<1^{\circ}\right)$. Proposed solutions to the "lunar inclination problem" invoke one or more subsequent excitation processes, including the evection and eviction resonances [74], Moonprotolunar disk resonant interactions [75], planetesimal scattering [76], or a Laplace plane instability [27]. Recently it has been recognized that inclination damping, as well as excitation, may have been important during the Moon's tidal evolution. In particular, large-scale inclination damping may have occurred at an Earth-Moon separation of 30 to 35 Earth radii when the lunar obliquity is expected to have experienced a large excursion [77, 27], particularly if the Moon's magma ocean had not solidified by this time. Improved constraints on when the magma ocean solidified would help constrain lunar orbital histories that in turn constrain origin models.

- Knowledge of lunar internal structure. Geochemical studies of lunar materials have suggested the existence of multiple mantle reservoirs [80,81], and geophysical studies have provided modest constraints on the size of the core $[82,83]$. Geophysical studies of the lunar interior can provide much needed constraints on the size and composition of the core, and possible deep mantle domains [84,85]. For example, the S content of the lunar core is not well constrained but is linked to the size and thermal state of the inner and outer core and will control the distribution of volatile and siderophile elements between core and mantle $[39,86]$. Linking geophysical and geochemical measurements will provide new constraints on the lunar thermal, magnetic, isotopic, and volatile evolution. Future seismic arrays on the lunar surface would place excellent constraints on the lunar internal structure.

- Modeling advancements. Modeling of giant-impact scenarios relies heavily on the equations of state of the materials involved in the impact, whose accurate description is necessary for calculating impact outcomes [78, 79]. Models of the evolution of the post-impact planet-disk system are also substantially under-constrained. In particular, direct links between the parameters that govern proto-lunar disk evolution and the chemical/isotopic composition of the resulting Moon are scarce. The development of new models that can constrain proto-lunar disk evolution via links with chemical/isotopic observables is needed to better understand the lunar origin process. 
Additionally, more realistic post-accretion lunar dynamical models are needed in order to distinguish between forming scenarios (e.g., the feasibility of AM removal mechanism that is required in some scenarios, or the evolution of the lunar inclination).

- We recommend that the decadal survey considers the critical role of team dynamics, equity, diversity, inclusion, and accessibility in planetary science. Studies of scientific teams have repeatedly demonstrated the importance of an integrated approach, where team members with diverse expertise and backgrounds develop synergies between their specialties and resources that result in an end product that adds up to more than the sum of its parts [88].

References: [1] Hartmann W.K. \& Davis D.R. (1975) Icarus 24, 504; [2] Cameron A.G.W. \& Ward W.R. (1976) LPSC VII; [3] Wood J.H. (1986) In Origin of the Moon, 17-56; [4] Benz W. et al. (1986) Icarus 66, 515; [5] Benz W. et al. (1989) Icarus 81, 113; [6] Cameron A.G.W. \& Benz W. (1991) Icarus 92, 204; [7] Cameron A.G.W. (1997) Icarus 126, 126; [8] Canup R.M. \& Asphaug E. (2001) Nature 412, 708; [9] Canup R.M. (2004) Icarus 168, 433; [10] Canup R.M. (2008) Icarus 196, 518; [11] Chambers J.E.(2001) Icarus 152, 2; [12] Pahlevan K. \& Stevenson D.J. (2007) Earth Plan. Sci. Let. 262, 438; [13] Wiechert U. (2001) Science 294, 5541; [14] Zhang J. et al. (2012) Nat.Geo, 5, 4; [15] Dauphas N. (2017) Nature 541, 521; [16] Pahlevan K. (2018) Nat. Geosci. 11, 16; [17] Cuk M. \& Stewart S.T. (2012) Science 338, 1047; [18] Lock S.J. et al. (2018) JGR Plan. 123, 910; [19] Canup R.M. (2012) Science 338, 1052; [20] Reufer A. et al. (2012) Icarus 221, 296; [21] Rufu R. et al. (2017) Nat. Geosci. 10, 89; [22] Belbruno E. \& Gott J.R. (2005) Astron. J. 129, 1724; [23] Touboul M. et al. (2015) Nature 520, 530; [24] Kruijer T.S. et al. (2015) Nature 520, 534; [25] Kruijer T.S. \& Kleine T. (2017) Earth Plan. Sci. Let. 475, 15; [26] Wisdom J. \& Tian Z. (2015) Icarus 256 138; [27] Ćuk M. et al. (2016) Nature 539, 402; [28] Tian Z. et al. (2017) Icarus 281, 90; [29] Ward W. R. et al. (2020) JGR-Planets, 125; [30] Tian Z. \& Wisdom J. (2020) PNAS 117, 27; [31] Rufu R. \& Canup R.M (2020), in review; [32] Citron R.I. et al. (2018) ApJ 862, 1; [33] Salmon J. \& Canup R.M. (2012) Ap. J. 760, 83; [34] Nakajima M. \& Stevenson D.J. (2018) Earth Plan. Sci. Let. 478, 117; [35] Paniello R.C et al. (2012) Nature 490, 376;[36] Wang K. \& Jacobsen S.B. (2016) Nature 538, 487; [37] Dhaliwa J.K. et al. (2018), Icarus 300, 249 [38] Dauphas N. et al. (2018), LPSC, 2083; [39] Righter K. (2019) Sci. Adv. 5, 1; [40] Barr A.C. (2016) JGR Plan. 121, 1573; [41] Andrews-Hanna J. et al. (2013) Science 339, 675; [42] Charlier B. et al. (2018) Geochim. Cosmochim. Acta 234, 50; [43] Lock S. J. \& Stewart S.T. (2019) Science Adv. 5, 9; [44] Pritchard M.E. \& Stevenson D.J. (2000) Origin of the Earth and Moon; [45] Solomon S.C (1977) Physics of the Earth and Planetary Interiors 15, 2-3; [46] Watters T.R. et al. (2010) Science 329, 5994; [47] Watters T.R. (2012) Nat. Geo 5, 3; [48] Lin Y.L et al. (2017) Nat.Geo 10, 1; [49] Khan A. et al. (2006) JGR-Planets 111; [50] Lognonné P. et al. (2003) Icarus, 211, 1-2; [51] Zhang N. et al. (2013), JGR-Planets, 118, 9; [52] Ringwood, A.E. \& Kesson, S. (1977) The Moon 16, 425; [53] Melosh H.J. et al. (2017) Geology, 45(12); [54] Charnoz S. \& Michaut C. (2015) Icarus, 260, 440; [55] Nie N.X. \& Dauphas N. (2019) ApJ 884, 2; [56] Melosh H.J (2014) Philos. Trans. R. Soc. A 372, 2024; [57] Canup R.M. et al. (2015) Nat.Geo. 8, 918; [58] Ward W.R (2012) ApJ 744, 140; [59] Ward W.R (2014) Philos. Trans. R. Soc. A 372, 2024; [60] Ward W.R (2017) JGR-Planets 122, 2; [61] Pahlevan K. et al. (2011) Earth Planet. Sci. Lett 301, 3-4; [62] Schiller M. et al. (2018), Nature 555, 7697; [63] Armytage et al. (2012), Geochim. Cosmochim. Acta 77, 504; [64] Warren P.H (2005) Meteorit. Planet. Sci. 40, 3; [65] Taylor S.R. (2006) Geochim. Cosmochim. Acta 70, 24; [66] Warren P.H. \& Dauphas N. (2014) LPSC 2298; [67] Dauphas N. et al. (2014) Philos. Trans. R. Soc. A 372, 2024; [68] Sakai R. (2014) Icarus 229, 42; [69] Dauphas N. \& Pourmand, A. (2011) Nature 473, 489 [70] Walsh K.J. et al (2012) Meteorit. Planet. Sci. 47, 12; [71] Clement M.S. et al. (2018) Icarus 311, 340; [72] Tsiganis K. et al. (2005) Nature 435, 7041; [73] Gomes R. et al. (2005) Nature 435, 7041; [74] Touma J. \& Wisdom J. (1998) Ap 115, 4; [75] Ward R.W \& Canup R.M (2000) Nature 403, 6771; [76] Pahlevan K. \& Mobidelli A. (2015) Nature 527, 7579; [77] Chen E.M.A \& Nimmo F. (2016) Icarus 275, 132; [78] Stewart S.T. et al. (2020) AIP Publishing, in press; [79] Hosono N. et al. (2019) Nat. Geo. 12, 418; [80] Robinson, K. L. \& Taylor, G. J. (2014) Nat. Geo 7, 6; [81] Cano E. J., et al. (2020) Nat. Geo 13, 4; [82] Garcia R.F. et al. (2011) PEPI 188, 96; [83] Weber, R.C. et al. (2011) Science 331, 309; [84] Righter K. et al. (2017) EPSL 463, 323; [85] Antonangeli D. et al. (2015) PNAS 112, 3916; [86] Steenstra E.S. et al. (2018) GCA 231, 130; [87] Nakajima, M., \& Stevenson, D. J., (2015) EPSL, 427, 286. [88] Balakrishnan A.D. et al. (2011) conference on Computer supported cooperative work 523; [89] Kokubo E. \& Genda H. (2010) Ap. J. Let. 714, L21; 